\title{
Using Genetic Algorithm to Determine the Optimal Portfolio
}

\author{
${ }^{1}$ Chien-Hua Wang, ${ }^{2}$ Wei-Hsuan Lee, ${ }^{2}$ Chia-Hsuan Yeh and ${ }^{3}$ Chin-Tzong Pang \\ ${ }^{1}$ School of Management, Fujian University of Technology, Fuzhou, China \\ ${ }^{2}$ Department of Information Management, Yuan Ze University, Taoyuan, Taiwan \\ ${ }^{3}$ Department of Information Management, and Innovation Center for Big Data and Digital Convergence, Yuan Ze University, Taoyuan, Taiwan
}

Correspondence Author: Chien-Hua Wang, School of Management, Fujian University of Technology, Fuzhou, China

E-mail: duraidaltaey@gmail.com

Received date: 22 January 2019, Accepted date: 14 April 2019, Online date: 25 April 2019

Copyright: (C) 2019 Chien-Hua Wang, Wei-Hsuan Lee, Chia-Hsuan Yeh, Chin-Tzong Pang., 2019. This is an open-access article distributed under the terms of the Creative Commons Attribution License, which permits unrestricted use, distribution, and reproduction in any medium, provided the original author and source are credited.

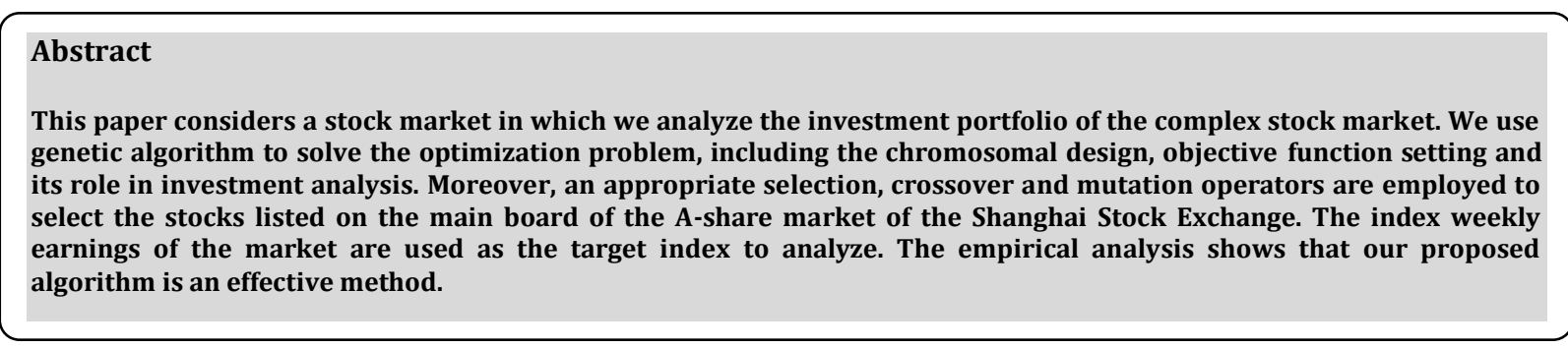

Keywords: Investment portfolio, Stock market, Genetic algorithm

\section{INTRODUCTION}

In recent years, as a major economic power in the world, China's economy has continued to develop and a large amount of capital has been invested in the Chinese financial markets. China's financial markets are complex and have various investment tools. Take the stock market as an example, there are numerous investment portfolios. However, financial markets are well-known places where imminent impacts are frequent, meaning risks and benefits coexist. Therefore, how to choose an appropriate investment portfolio within a limited time has become a challenge for each investor (Chen \& Xiao, 2003). Since the 1940s, the algorithms which mimics biological behavior have become the way of scientific computing (Statman, 1999). According to Darwin's theory of evolution, the genetic algorithm (GA) for simulating natural evolution provides a powerful method for computational optimization and provides an effective method to resolve large-scale complex computations. The advantage of GA is that it simulates the biological evolution which has been shown to be an effective way to solve the optimization problem. In addition, a satisfactory solution can be obtained under a lower cost in terms of time and computational complexity. Therefore, GA is widely employed as a tool to solve the theoretical as well as empirical optimization issues.

The main analysis of stock market regarding forecasting consists of two types. One is the fundamental analysis. Using the information of market supply and demand to determine the fundamental prices so as to provide decision-making reference for investors. Besides, the long-term forecasting about individual stock is obtained through the analysis of the macro information of the market, industry, and company. The other is technical analysis. This method uses technical indicators to examine the patterns of price dynamics and price trends based on historical data. In other words, the fundamental analysis pays attention to the longterm perspective of fundamental information and values of stocks, while the technical analysis is conducted based on the shortterm behavior of prices. However, forecasting based on both methods simultaneously is not an easy job. This paper tries to employ GA to resolve this problem through two steps. First, we use the fundamental analysis to remove the stock with poor performance. Second, GA is used to select an appropriate stock portfolio.

\section{LITERATURE REVIEW}

Investment portfolio: The investment portfolio has two meanings. One is to select investment products among the stocks with similar characteristics. It means that the different combinations of stocks result in different investment performance. The other is the funds diversified in different types of investment products. For example, investing in stocks and funds at the same time can 
reduce the risk. In short, the core concept of an investment portfolio is to use the method of portfolio to diversify the investment funds and risk so as to maximize benefits and minimize the risks. (Zhao \& Wang, 2015). In 1952, Markowitz explained the central concept of modern portfolio theory.

Under the assumption that the investors select rational investment portfolio and maximize their utility (Markowitz, 1952), the utility of the investor is a function about the expected returns and standard deviation of the portfolio. A rational investor always seeks the highest returns in affordable risks or to minimize risks at an acceptable level of returns.

In summary, the modern investment theory is about investment combinations. One is to minimize risk under a given return, the other is to maximize returns under a given risk. Investors with different risk preferences will select different methods of investment. The main goal is to diversify investment funds and reduce investment risks simultaneously to improve investment returns.

Genetic algorithm: Genetic algorithm (GA) is an optimization and searching technique based on the principles of genetics and natural selection. It was developed by Holland and his colleagues in 1975 (Haupt \& Haupt, 2004). The basic concept of GA is the natural selection, survival of the fittest and natural evolution in biology, in which parents with better fitness exchange genetic information, so that the offspring may possess better performance compared with that of their parents. Each iteration process for GA is called a generation. The entire set of generations is called a run. In GA, a chromosome refers to a candidate solution to a problem and is often encoded as a bit string. The genes can be single bits or short blocks of adjacent bits that encode a particular element of the candidate solution. Crossover typically consists of exchanging genetic material between two chromosomes from parents. The crossover operator randomly chooses the crossover point and then exchanges the chromosome parts after that point. Then two new offspring are created. If a pair of chromosomes do not execute the crossover operator, then the reproduction is executed instead. In other words, the resulting offspring is the same as the parents. The mutation represents a change in the gene, and this rarely happens in nature. The aim of mutation is to make a significant improvement in fitness. The mutation operator also protects the search algorithm from trapping on a local optimum. The variation is also known as a random search with excellent parallel processing capabilities and it can help in avoiding loss of genetic diversity. Therefore, GA can simultaneously search for many feasible solutions and obtain solutions with both efficiency and effectiveness based on the design of various computing conditions of decision-makers (Ji, 2004).

\section{RESEARCH DESIGN}

The section introduces the content of GA. The definition of chromosome and introduction of fitness function are described in Section 3.1. Section 3.2 details the coding scheme and genetic operations.

Definition of chromosome: We consider the content of GA as the stock code and indicators. A gene represents a stock. The combination of the genes forms a chromosome which means a stock portfolio. In this paper, a binary encoding is used for encoding, namely 0-1 encoding. Consider a portfolio with 20 stocks which are chosen from n stocks, then there are $\mathrm{C}_{20}^{\mathrm{n}}$ possible chromosomes. That is, the size of sample space is $\mathrm{C}_{20}^{\mathrm{n}}$. Next, the fitness function used in this paper consists of four parts, daily return (Dret), tradable shares daily turnover ratio (DTrdTurnR), beta_tradable market value-weighted (Beta_tmv) and KDJ index, respectively. Thus, the formula of fitness function is defined as

$$
\begin{aligned}
& f\left(\text { Genen }_{i}\right)=w_{1} \cdot \text { Dret }+w_{2} \cdot \text { DTrdTurnR }+ \\
& w_{3} \cdot \text { Beta_tmv }+w_{4} \cdot K D J
\end{aligned}
$$

where $w_{1}, w_{2}, w_{3}$ and $w_{4}$ are the corresponding weight values. The fitness function of a chromosome is:

$$
f\left(\text { Chm }_{i}\right)=\frac{\left(\sum f\left(\text { Genen }_{j_{i}}\right)\right)}{n}
$$

where $n$ is the number of chromosomes.

Genetic operations: The roulette method is used as a selection mechanism because individuals with high fitness will have greater opportunities as parents and transmit genes to the next generation. In addition, in the crossover operation, multi-point crossover is applied. There are eight crossover points in total. The first crossover point is the location of the stock, and the other seven points are the daily yield of the stock. The Beta value divides the stock into eight points, the first four crossover points are set as stocks with Beta value less than one, and the last four points are set as stocks with a Beta value greater than one. Moreover, for excellent parent individuals, their substrings are exchanged. The resulting offspring with the highest fitness value is selected and compared with its parents. Two chromosomes with most senior fitness are then selected as the candidate solutions in the next generation (Yang \& Wang, 2009). 
The probability of cross $=($ The number of chromosomes above the average fitness value / Population size) $\times \theta$

where $\theta$ is the adjustment coefficient.

In the setting of mutation operation, a chromosome from the population is randomly selected and it is replaced with a new one, and then mutation operator is applied to this new one individual. The formula is as follows.

The probability of mutation $=$ (current generation - genetic generation) / genetic generation $\times \theta$

where the upper limit is equal to 0.01 .

As for the setting of the termination condition, it is mainly determined by the degree of fitness of the population. By calculating the fitness of each chromosome of a population, the algorithm will stop if it meets certain requirements or the stagnation of the fitness changes.

\section{Empirical results}

The data is obtained from RESSET's stock database. The relevant indicators of the data are listed as follows (all stocks the Ashares of the Shanghai Stock Exchange).

Daily Stock Comprehensive Data (2016.7.1 - 2016.7.31). Stock code,_Stkcd;

Daily Stock Comprehensive Data (2016.7.1 - 2016.7.31). Latest stock name,_Lstknm;

Daily Stock Comprehensive Data (2016.7.1 - 2016.7.31). Date,_Date;

Daily Stock Comprehensive Data (2016.7.1 - 2016.7.31). Highest price,_Hipr;

Daily Stock Comprehensive Data (2016.7.1 - 2016.7.31). Lowest price,_Lopr;

Daily Stock Comprehensive Data (2016.7.1 - 2016.7.31). Closing price,_Clpr;

Daily Stock Comprehensive Data (2016.7.1 - 2016.7.31). Exchange rate of daily shares,_DTrdTurnR;

Daily Stock Comprehensive Data (2016.7.1 - 2016.7.31). Daily rate of return, _Dret;

Financial indicator (2016.7.1 - 2016.7.31). Net profit,_Netprf;

Financial indicator (2016.7.1 - 2016.7.31). Total assets,_Totass;

Financial indicator (2016.7.1 - 2016.7.31). Total liabilities,_Totlia;

Using the above-mentioned data, we perform the first step calculation. The data including the following indexes,_Stkcd,_Dtrd TurnR,_Dret,_K21, D21, J21 and_Beta, a total of 1095 stocks, and 19612 data, are obtained. Using the information obtained from the first step, the stocks of all the A-shares listed on the Shanghai Stock Exchange that are listed and whose time-to-market is greater than 3 months are excluded from the initial sample space ( $\mathrm{Hu}$, Pang \& $\mathrm{Hu}, 2003$ ).

(1) The stocks for company name changed;

(2) The stocks for securities code changes;

(3) The stocks for net assets are negative;

(4) The stocks with negative net profits;

(5) The stocks for net investment profit with negative.

After excluding above-mentioned illegal stocks, 465 stock are obtained, and then the stocks that are difficult to obtain complete data are eliminated. Finally, 413 stocks are selected as alternative stocks. The final alternative stocks and related data are as follows (a total of 413 data, only the top 20 are list here)

Table 1: The data of alternative stock.

\begin{tabular}{|c|c|c|c|c|c|c|}
\hline Stkcd & DTrdTurnR & Dret & K21 & D21 & J21 & Beta \\
\hline 600004 & 0.701461905 & 0.004980952 & 70.48006194 & 60.21422119 & 91.01174345 & 1.011461905 \\
\hline 600006 & 2.229271429 & -0.005842857 & 32.59871442 & 42.11815121 & 13.55984083 & 1.293057143 \\
\hline 600007 & 0.206666667 & 0.00387619 & 74.56474589 & 61.82668883 & 100.04086 & 1.040366667 \\
\hline 600023 & 0.97682381 & 0.00077619 & 48.69281046 & 49.78213508 & 46.51416122 & 1.240728571 \\
\hline 600031 & 0.395319048 & 0.001838095 & 48.30917874 & 49.59742351 & 45.73268921 & 1.160004762 \\
\hline 600037 & 2.637742857 & 0.005080952 & 61.99494949 & 56.18686869 & 73.6111111 & 1.438685714 \\
\hline 600050 & 0.537742857 & 0.005547619 & 63.76811594 & 57.32689211 & 76.65056361 & 1.10232381 \\
\hline 600051 & 1.860738095 & -0.000842857 & 26.91511387 & 39.1994479 & 2.346445825 & 1.342004762 \\
\hline 600056 & 1.082304762 & 0.005128571 & 57.69091567 & 53.95101942 & 65.17070817 & 1.299952381 \\
\hline
\end{tabular}


Citation: Chien-Hua Wang, Wei-Hsuan Lee, Chia-Hsuan Yeh, Chin-Tzong Pang, 2019. Using Genetic Algorithm to Determine the Optimal Portfolio Advances in Natural and Applied Sciences., 13(1): 1-6. DOI: 10.22587/anas.2019.13.1.1

\begin{tabular}{|c|c|c|c|c|c|c|}
\hline 600057 & 1.279061905 & 0.002285714 & 52.63584753 & 52.04109219 & 53.8253582 & 1.35477619 \\
\hline 600058 & 2.46822381 & -0.002628571 & 26.22222222 & 39.42592593 & -0.185185185 & 1.450547619 \\
\hline 600060 & 1.215628571 & 0.001633333 & 51.01654846 & 50.32308905 & 52.4034673 & 1.399519048 \\
\hline 600061 & 1.026071429 & -0.003233333 & 25.88075881 & 39.1598916 & -0.677506775 & 1.558852381 \\
\hline 600062 & 1.604914286 & 0.006747619 & 71.14338456 & 59.63293032 & 94.16429302 & 1.178571429 \\
\hline 600064 & 1.29117619 & 0.006061905 & 69.82873958 & 58.85195829 & 91.78230215 & 1.287133333 \\
\hline 600066 & 0.670019048 & 0.007209524 & 68.98281056 & 59.78439364 & 87.3796444 & 0.780380952 \\
\hline 600067 & 0.861366667 & -0.002419048 & 26.70250896 & 39.84468339 & 0.418160096 & 1.41417619 \\
\hline 600073 & 2.728533333 & 0.005095238 & 64.12988331 & 56.95924235 & 78.47116523 & 1.282490476 \\
\hline 600075 & 3.7532 & 0.005471429 & 67.10695947 & 59.24141447 & 82.83804946 & 1.392152381 \\
\hline 600076 & 3.25347619 & -0.003733333 & 31.4957265 & 41.38176638 & 11.72364672 & 1.324371429 \\
\hline
\end{tabular}

where Stkcd represents the stock code, DTrdTurnR represents the daily stock exchange rate, Dret represents the daily yield, K21 represents the $21^{\text {st }} \mathrm{K}$ value, D21 represents the $21^{\text {st }} \mathrm{D}$ value, $\mathrm{J} 21$ represents the $21^{\text {st }} \mathrm{J}$ value, and Beta represents the $\beta$ coefficient.

The next step is to perform binary processing. The processing rule is to take the integer part of the data for the first binary, then measures the length of the digitized digit in the binary, and takes the longest digit as the number of bits to perform the binary calculation again. After calculation, the longest binary field is 10 bits long, so all data is converted into binary data with a length of 10 characters. Each unit of the encoded data is a gene segment, and genes are generated in the order of 7 gene segments now. The 10 bits in the head of each gene are the sequence numbers, of this gene, and a total of 413 genes will be generated. The chromosome is the combination of the above genes. The final population set is 20 , and $\mathrm{C}_{20}^{413}$ chromosomes will be generated here. In addition, this paper is performed based on the Matlab software, each takes 51 generations, and the comprehensive conditions of the obtained indicators are as follows (Lee, 2013).
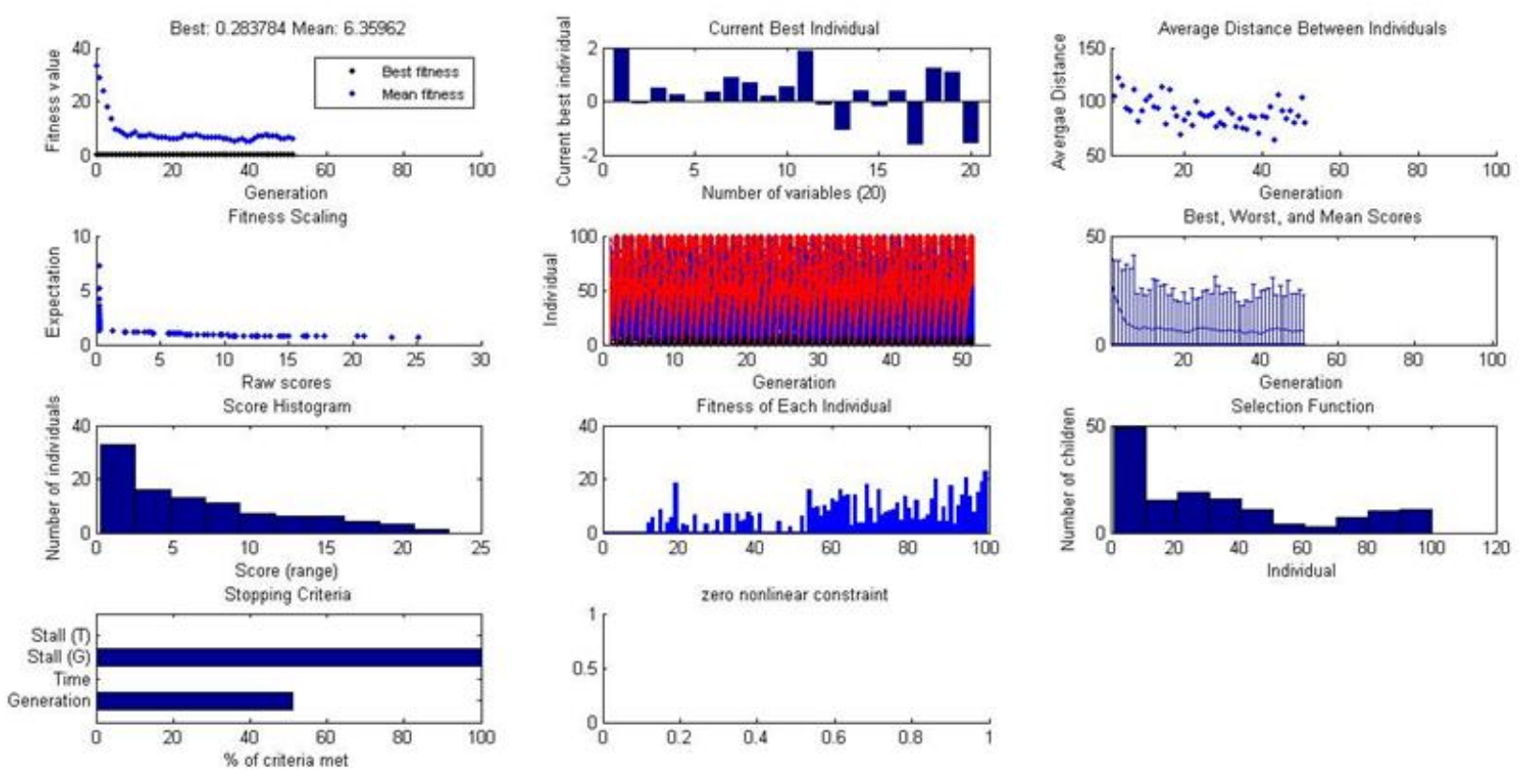

Fig. 1: Comprehensive state of each index

In Fig. 1, it is observed that the optimum fitness is 0.283783 . And 20 stocks and their data corresponding to the calculation rules are as follows.

Table 2: The data of alternative stock

\begin{tabular}{|c|c|c|c|c|c|c|}
\hline num & DTrdTurnR & Dret & K21 & D21 & J21 & Beta \\
\hline 13 & 10.26071429 & -16.16666667 & 25.88075881 & 39.1598916 & -0.677506775 & 155.8852381 \\
\hline 22 & 19.34885714 & -4.142857143 & 34.30555556 & 43.7037037 & 15.50925926 & 158.4342857 \\
\hline 64 & 46.13214286 & -42.92857143 & 25.27632344 & 38.75315106 & -1.677331782 & 158.6319048 \\
\hline 115 & 5.59352381 & 5.214285714 & 59.2039801 & 54.22885572 & 69.15422886 & 136.5280952 \\
\hline 120 & 19.45066667 & -34.66666667 & 29.02322978 & 40.3675716 & 6.334546133 & 153.0104762 \\
\hline
\end{tabular}


Citation: Chien-Hua Wang, Wei-Hsuan Lee, Chia-Hsuan Yeh, Chin-Tzong Pang, 2019. Using Genetic Algorithm to Determine the Optimal Portfolio Advances in Natural and Applied Sciences., 13(1): 1-6. DOI: 10.22587/anas.2019.13.1.1

\begin{tabular}{|l|c|c|c|c|c|c|}
\hline 142 & 8.84552381 & -11.54761905 & 46.49202048 & 48.70019071 & 42.07568002 & 133.837619 \\
\hline 145 & 26.114 & -32.69047619 & 25.92592593 & 38.73097904 & 0.315819696 & 147.1752381 \\
\hline 180 & 37.15009524 & -39.54761905 & 27.7179879 & 39.72509144 & 3.703780802 & 173.7485714 \\
\hline 181 & 29.84852381 & -50.83333333 & 24.56294511 & 38.19771886 & -2.706602389 & 150.1404762 \\
\hline 185 & 13.25752381 & -37.76190476 & 26.5795207 & 39.50617284 & 0.726216412 & 137.4147619 \\
\hline 231 & 15.60314286 & 2.761904762 & 33.38041431 & 43.4871312 & 13.16698054 & 163.797619 \\
\hline 239 & 10.34795238 & 16.0952381 & 62.35167206 & 56.6702625 & 73.71449119 & 148.5961905 \\
\hline 242 & 5.67747619 & -2.30952381 & 47.39583333 & 48.78472222 & 44.61805556 & 146.0890476 \\
\hline 261 & 18.6527619 & -41.57142857 & 33.31904252 & 42.01262356 & 15.93188043 & 122.9519048 \\
\hline 276 & 27.02833333 & -0.976190476 & 44.28341385 & 48.04079442 & 36.76865271 & 158.1061905 \\
\hline 300 & 5.102285714 & 11.88095238 & 63.44605475 & 58.07836822 & 74.1814278 & 144.4457143 \\
\hline 311 & 4.768571429 & 10.23809524 & 57.52688172 & 53.22580645 & 66.12903226 & 136.4580952 \\
\hline 319 & 3.973047619 & 8 & 56.89655172 & 54.59770115 & 61.49425287 & 137.6509524 \\
\hline 335 & 33.71819048 & -35.30952381 & 28.13051146 & 40.18224574 & 4.027042916 & 148.4757143 \\
\hline 397 & 10.73642857 & -2.976190476 & 36.68981481 & 44.25154321 & 21.56635802 & 153.9942857 \\
\hline
\end{tabular}

Moreover, in Aug., the predicted portfolio return is compared with the market daily revenue, as shown in Table 3.

Table 3: The data of alternative stock

\begin{tabular}{|c|c|c|}
\hline Date & Market daily rate of return & Combined prediction daily rate of return \\
\hline $2016-08-01$ & -0.0079 & -0.01683 \\
\hline $2016-08-02$ & 0.0059 & 0.011495 \\
\hline $2016-08-03$ & 0.0017 & 0.0051 \\
\hline $2016-08-04$ & 0.0012 & 0.007925 \\
\hline $2016-08-05$ & -0.001 & -0.007445 \\
\hline $2016-08-08$ & 0.0093 & 0.0193 \\
\hline $2016-08-09$ & 0.0071 & 0.0091 \\
\hline $2016-08-10$ & -0.0025 & 0.000135 \\
\hline $2016-08-11$ & -0.004 & -0.01486 \\
\hline $2016-08-12$ & 0.0167 & 0.016485 \\
\hline $2016-08-15$ & 0.0247 & 0.035925 \\
\hline $2016-08-16$ & -0.0059 & 0.009505 \\
\hline $2016-08-17$ & -0.0001 & -0.00125 \\
\hline $2016-08-18$ & -0.0016 & -0.001385 \\
\hline $2016-08-19$ & 0.0021 & 0.007635 \\
\hline $2016-08-22$ & -0.0069 & -0.00371 \\
\hline $2016-08-23$ & 0.0017 & -0.003147368 \\
\hline $2016-08-24$ & -0.0015 & -0.001231579 \\
\hline $2016-08-25$ & -0.0059 & -0.012668421 \\
\hline $2016-08-26$ & 0.0005 & 0.00033 \\
\hline $2016-08-29$ & -0.0001 & -0.00274 \\
\hline $2016-08-30$ & 0.0021 & 0.00208 \\
\hline $2016-08-31$ & 0.0034 & 0.00664 \\
\hline
\end{tabular}

The result shows that the predicted daily return of the portfolio can well track the daily return of the market index. The average daily market yield of the A-share motherboard market in Aug. 2016 is 0.001695652, and the average daily return of the predicted stock portfolio is 0.002886419 . In addition, in Aug. 2016, the daily market return of the A-share motherboard market is 0.0390, and the daily yield of the predicted stock portfolio adds to 0.0664 . Consequently, the calculated return of the portfolio is $70.22 \%$ higher than the A-share motherboard market.

\section{CONCLUSIONS}

Portfolio theory is a theory accepted and approved by the general public. By dispersing funds and diversifying investment risks, it can obtain considerable investment returns under acceptable risks. Based on the A-share motherboard market of the Shanghai Stock Exchanges, this paper adopts GA to solve the optimization problem for the portfolio.

The results show that the daily return of the portfolio obtained by GA can well track the daily return of the corresponding market, and the overall yield of the solution is $70 \%$ higher than the market return. Therefore, the method designed in this paper is effective 
for portfolio optimization. In addition, compared with the exhaustive search for all solutions, GA can use less resource in terms of computational time for producing satisfactory results. In the future, we will attempt to design different methods of GA with various problem domains.

\section{REFERENCES}

[1] Chen, K. Y. \& Xiao, D. R, 2003. Study of optimal models for portfolio investment based on genetic algorithm, Journal of Nanjing Institute of Meteorology, 26(5): 707-711.

[2] Haupt, R. L. \& Haupt, S. E., 2004. Practical genetic algorithms. New Jersey: John Wiley \& Sons, Inc.

[3] Hu, B, Pan, F. Z. \& Hu, Q. F., 2003. Application of the genetic algorithm is short-term stock investment decision making. Systems Engineering- Theory \& Practice, 23(2): 7-13.

[4] Ji, G. L., 2004. Survey on genetic algorithm. Computer application and Software, 21(2), 69-73.

[5] Lee, K. M, 2013, Arbitrage analysis of stock index futures based on the genetic algorithm for optimizing the tracking error. Journal of Yunnan Minzu University (Nat. Sci. Edition), 22(5): 349-354.

[6] Markowitz, H., 1952. Portfolio selection. Journal of Finance. In: Journal of Finance, 7(1): 77-91.

[7] Statman, M., 1999. Behaviorial finace: pastbattles and engagements. Financial Analysis Journal, 55(6): 18-27. Yang, G. Y. \& Wang, X., 2009. Investment decision- making of portfolio based on genetic algorithm. Science Technology and Industry, 9(3): 44-49.

[8] Zhao, Q. \& Wang, Z. Q., 2015. A summary of the practical application of portfolio theory and its development trend. Journal of Zhe Jiang Gong Shang University, 1(130), 82-91. 